\title{
Utility of large sections (macrosections) in breast cancer pathology
}

\author{
Melissa A. T. Monica, Luca Morandi, Maria P. Foschini \\ Department of Biomedical and Neuromotor Sciences, Section of Anatomic Pathology at Bellaria Hospital, University of Bologna, Bologna, Italy \\ Contributions: (I) Conception and design: MA Monica, MP Foschini; (II) Administrative support: MP Foschini; (III) Provision of study materials \\ or patients: All authors; (IV) Collection and assembly of data: MA Monica, L Morandi; (V) Data analysis and interpretation: All authors; (VI) \\ Manuscript writing: All authors; (VII) Final approval of manuscript: All authors. \\ Correspondence to: Prof. Maria P. Foschini, MD. Section of Anatomic Pathology, Bellaria Hospital, Via Altura 3, Bologna 40139, Italy. \\ Email: mariapia.foschini@unibo.it.
}

\begin{abstract}
Large sections (LSs), (synomim: macrosections), are used in daily routine practice of breast pathology to better investigate the disease and to allow a thorough diagnosis. It has been demonstrated that costs do not differ from conventional blocking and the turn-around-time could be no longer than 24 hours. Immunohistochemistry or molecular studies are feasible by selecting areas of interest from LS and cutting them to obtain small conventional blocks. LSs are also employed in surgical pathology of various organs, among which breast is comprised. LS in breast pathology is useful to a proper definition of stage disease, tumor size (TS), tumor extent (TE), tumor multifocality (TM) and resection margins. The presence and extension of in situ carcinoma is more completely observed, overcoming the difficulties of gross inspection at naked eye. In the multidisciplinary era, LSs allow the correlations between radiological and pathological data. In addition, LSs play an important role in cases treated with neo-adjuvant (NAD) chemotherapy, as they are useful to better evaluate the residual cancer burden and the degree of tumour regression. The aim of the present paper is to review the value of LS in breast pathology.
\end{abstract}

Keywords: Large section (LS); histology; breast cancer; carcinoma in situ; invasive carcinoma; resection margin; tumor extent (TE); tumor multifocality (TM)

Submitted May 30, 2017. Accepted for publication Jun 26, 2017.

doi: $10.21037 /$ tcr.2017.08.12

View this article at: http://dx.doi.org/10.21037/tcr.2017.08.12

\section{Introduction}

Large sections (synonim: macrosections, large format sections) (LSs) consist of an entire histological section of the organ under investigation. Tissue obtained by LS encompasses not only the lesions, but also the surrounding tissue, the resection margins and allows radiologicalpathological correlation useful to a better understanding of the diseases. The aim of the present paper is to review the basis of applications of LS, their current use in breast pathology, and to provide an overview on the history and applications of this technique.

\section{History of LSs in breast pathology}

The first use of LSs in breast pathology was reported by Cheatle at the beginning of the $20^{\text {th }}$ century (1-3), who aimed to study the relation between breast cancer and the surrounding tissue.

Subsequently, several studies based on LS were performed leading to the hypothesis that breast cancer originate mainly from the terminal ductular-lobular unit (TDLU) (4). Specifically in 1973, Wellings and Jensen analyzed cases of in situ and invasive ductal and lobular carcinomas, leading to the proposal that most breast carcinomas arise in the TDLU (4). The relation between breast cancer and surrounding normal tissue was further studied in 1939 by Ingleby and Holly (5). Marcus and Welling improved a method to study early phases of breast cancer development (6-8). The application LS in breast pathology helped in highlighting the importance of multiple invasive tumor deposits (multifocality). Sarnelli and Squartini (9) and Tot $(10,11)$ demonstrated that multifocal breast carcinomas carry a worse prognosis 


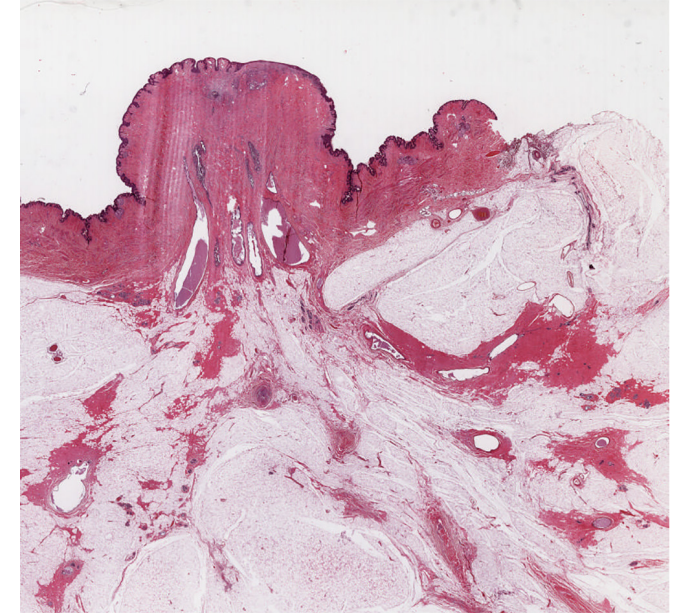

Figure 1 Breast normal tissue, comprising the nipple and galactophorous ducts. The LS allows to retrace the ducts penetrating into the breast parenchyma (magnification $1 \times$ ). LS, large section.

when compared to unifocal lesions. The extent and type of growth of different types of ductal carcinomas in situ (DCIS) had been investigated with LS since they proved their effectiveness in better visualizing distribution of lesions not evident at gross examination (11,12). In 1992, Faverly et al. (13) reconstructed a three-dimensional glandular tree of the breast, evaluating the extent and distribution of DCIS. This led to the assumption that DCIS is a multifocal process and that multifocality is characteristic of low grade rather than high grade DCIS. In more recent years, LSs were useful to better understand the breast microanatomy and to assess radiological-pathological correlations. Going and Moffat $(14,15)$ published detailed studies on the breast microanatomy, describing the threedimensional reconstruction of the breast parenchyma and its organization in "lobes" each one belonging to a galactophorous duct (Figure 1). In the same years, Tot and Tabar applied the LS technique to obtain pathological radiological correlations, that lead to a better understand of the various radiological pictures encountered in screen mammography programs $(16,17)$. More recently the same radiological-pathological correlations were applied to images obtained by nuclear magnetic resonance $(16,17)$.

All these data lead Tot $(10,18)$ to propose the theory of the "sick lobe" as the basis of breast cancer development and growth. According to Dr. Tot's hypothesis, breast cancers originate from the epithelium of a single ductal system, the sick lobe, in which epithelial cells undergo malignant transformation. As a consequence of the sick lobe theory, breast carcinoma grows along the branches of a single lobe. Data obtained by Going and Moffat (14) indicate that size of each breast lobe varies greatly. Therefore, it is not unexpected that the extent of breast cancer can be extremely variable according to the type and size of the affected lobe.

\section{Methods}

Methods to obtain LSs have been described in detail in several papers (18-20). The most important clues to follow, in order to obtain LS useful in routine diagnoses, are related to specimen cutting. Surgical specimens should be received oriented by the surgeon and possibly compared with the radiological imaging. Cutting should be performed with proper large blade according to the radiological images. In our institution, surgical specimens are sliced into sections ranging from 5 to $10 \mathrm{~mm}$ in thickness. The number of LS obtained varies in each case. In breast specimens surgically resected after neo-adjuvant (NAD) chemotherapy with absence of residual macroscopic tumor, LS are utilized to completely embed the tumor bed. In cases of quadrantectomies, whose larger dimension is less than $5 \mathrm{~cm}$, the entire specimen can be embedded, according to the same procedures applied in prostatectomies, as recommended by Montironi et al. (21). The costs and turnaround time (TAT) of LS in daily routine practice have been calculated and compared to conventional blocks. The final LS costs and TAT do not substantially differ from those of conventional block $(22,23)$. When immunohistochemistry or molecular studies are required, small conventional blocks can be obtained by selecting the area of interest.

\section{Application of LS in breast pathology}

\section{Tumor size (TS) (Figure 2)}

TS is at the basis of the TNM classification system, and is considered one of the most powerful prognostic indicator in tumor pathology. Therefore, TS correct evaluation is of outmost importance in daily practice. The superior value of LS in the correct TS evaluation was originally demonstrated by Jackson et al. (24) who compared two series of breast cancers, one staged with conventional blocks and one staged with LS. According to Jackson et al.'s paper, the correct TS was obtained in $63 \%$ of the conventional block series and in all cases of the LS series. In addition, LS are especially helpful in cases of invasive lobular carcinoma (21).

\section{Tumor extent (TE) (Figure 3)}

Invasive carcinoma, both of ductal and lobular type is 


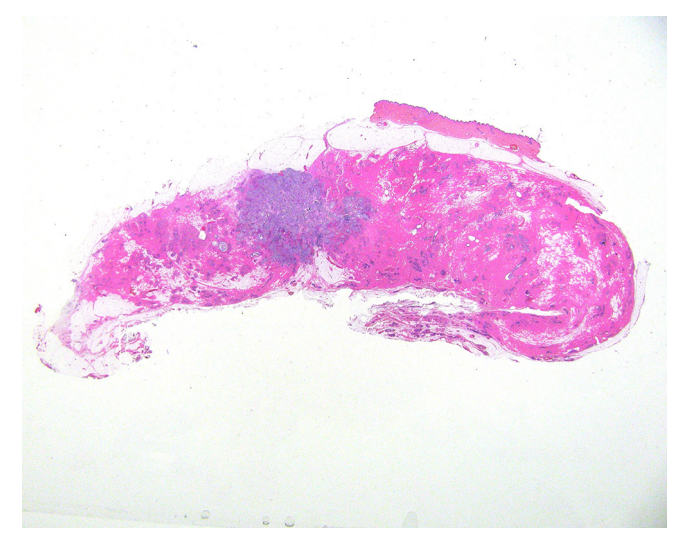

Figure 2 LS with large neoplastic area and the surrounding tissue. The LS allows to correctly evaluate the tumor size (magnification $1 \times)$. LS, large section.

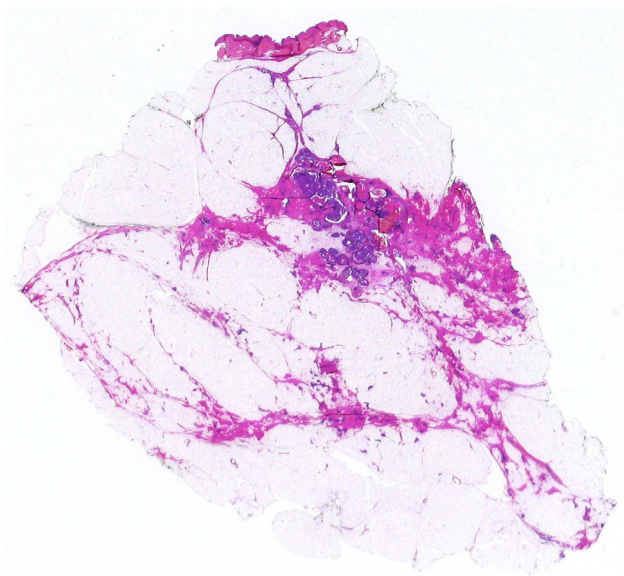

Figure $3 \mathrm{TE}$ can be easily evaluated in this large section of low grade DCIS (magnification $1 \times$ ). TE, tumor extent; DCIS, ductal carcinoma in situ.

frequently surrounded by the in situ counterpart. It has been demonstrated that the total extent of the neoplastic area, composed both by the in situ and invasive components (TE), has an important prognostic impact. TE $>40 \mathrm{~mm}$ is associated with higher risk of local recurrence after breast conserving surgery, and to shorter disease free survival (25). $\mathrm{TE}$ is related also to the type of growth of the breast cancer. Diffuse invasive carcinomas, characterized by a diffuse type of growth, similar to a "spider web", carry a higher risk of recurrence and disease progression (26).

\section{Tumor multifocality (TM) (Figure 4)}

The incidence of TM varies greatly, based on the method

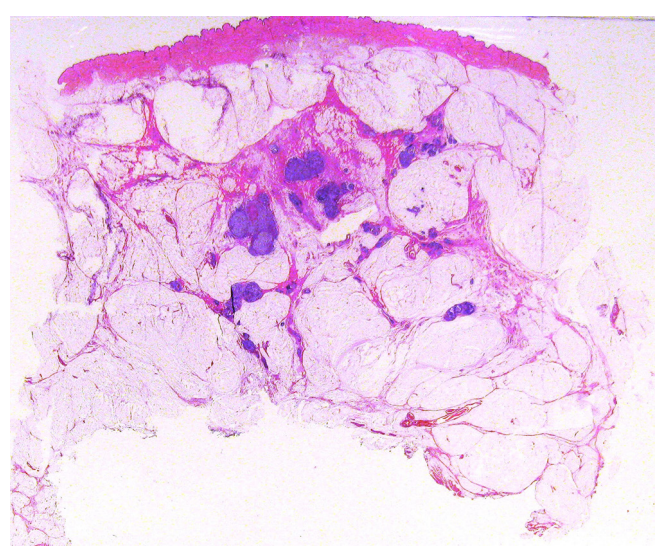

Figure 4 LS allows the detection of small tumor foci, helping in the evaluation of tumour multifocality (magnification 1x). LS, large section.

of detection (27), as a consequence its prognostic impact has been debated for a long time. Earlier papers, based on conventional blocks, denied the prognostic importance of TM.

These uncertainties and discussions were consequent to unclear definition of TM. Tot proposed an easily reproducible definition, that is to consider separate neoplastic foci, when they are separated by non-neoplastic glandular tissue $(11,12)$. This definition has been validated on molecular grounds, demonstrating that the tumoral foci, when separated by non-neoplastic glandular structures, are not clonally related (28). The introduction of modern radiological techniques has led to the more frequent discover of TM (29), therefore it is mandatory for pathologists the use of LS that allow a good correlation with radiological findings and an accurate detection of all neoplastic foci. Multiple neoplastic foci can be related both to in situ and invasive carcinoma. Studies based on LS (30) demonstrated that multiple foci of invasive carcinomas are related to a worse prognosis. Nevertheless, the dismal prognostic impact of TM can be improved, by proper treatments (31). Therefore, TM should always be searched in all cases of breast cancer.

\section{Resection margins (Figure 5)}

Evaluation is important to reduce the local recurrence rate and to correctly plan post-surgical therapy. The presence of in situ carcinoma surrounding the tumor is one of the most frequent causes of positive resection margin. Routinely application of LS to breast conserving surgical specimens, allows a correct evaluation of in situ carcinoma extent. In addition, when LS are oriented according to the surgeon's instructions, it is possible to evaluate which resection 
margin is involved by the tumour (28).

\section{Post-NAD evaluation (Figure 6)}

Complete pathological response to NAD chemotherapy is related to better prognosis, both in terms of overall survival and of disease-free survival (32). It is not infrequent that cases showing complete pathological response on radiological imaging, can still present minute neoplastic foci in the tumor bed $(33,34)$. Therefore, pathological evaluation of the entire tumor bed, is of crucial importance to properly treat the patients. To this purpose the tumor bed area should be completely embedded and LS constitute an ideal tool.

\section{Discussion}

The pathology report contains information, such as TS,

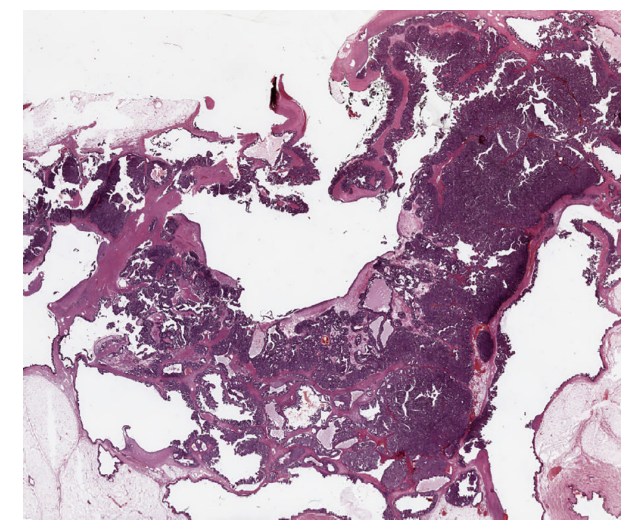

Figure 5 The present LS illustrates a large papillary carcinoma. The relation with resection margin is clearly seen (magnification $1 \times)$. LS, large section.
TE, TM and margins status, that significantly influence the therapeutic planning. LS are of certain utility in defining essential aspects that need to be evaluated in breast cancer. First of all, LSs allow the comparison between radiological imaging and specimen obtained from surgery. The preoperative study is made possible by mammography and magnetic resonance imaging. During mammography, the breast is compressed, while in magnetic resonance imaging the antero-posterior axis become transiently longer than in the up-right position (26), so that measurement of TS could be partially inaccurate. The pathologist can analyze a case starting from the radiological orientation, and, if possible, the specimen should be subsequently radiographed. In a single institution report, LS histology categories of TS and disease extent were concordant with radiological measurements in approximately $80 \%$ of the cases. Non-calcifying, low-grade in situ foci and invasive tumor foci $<5 \mathrm{~mm}$ are the most frequent causes of discrepant findings with histopathological examination (25). LS allows a better definition of pathologic stage, known as the most powerful prognostic parameter. TS is defined as the largest diameter of the invasive tumor focus and is a constituent of TNM staging system (25). Breast cancers show irregularity in shape, and LS improve the accuracy of measurement. In addition, using LS, the pathologist can easily verify also the presence of intratumoral heterogeneity (17). With conventional blocking, the specimens undergo fragmentation in samples measuring $1-2 \mathrm{~cm}$ and the three-dimensional structure is necessarily lost. The preservation of breast anatomy is an essential aspect LS (19). Subgross lesion distribution and disease extent carry prognostic information as a diffuse or multifocal invasive component is associated with an increased risk of cancer-related death, compared with unifocal tumours (30).
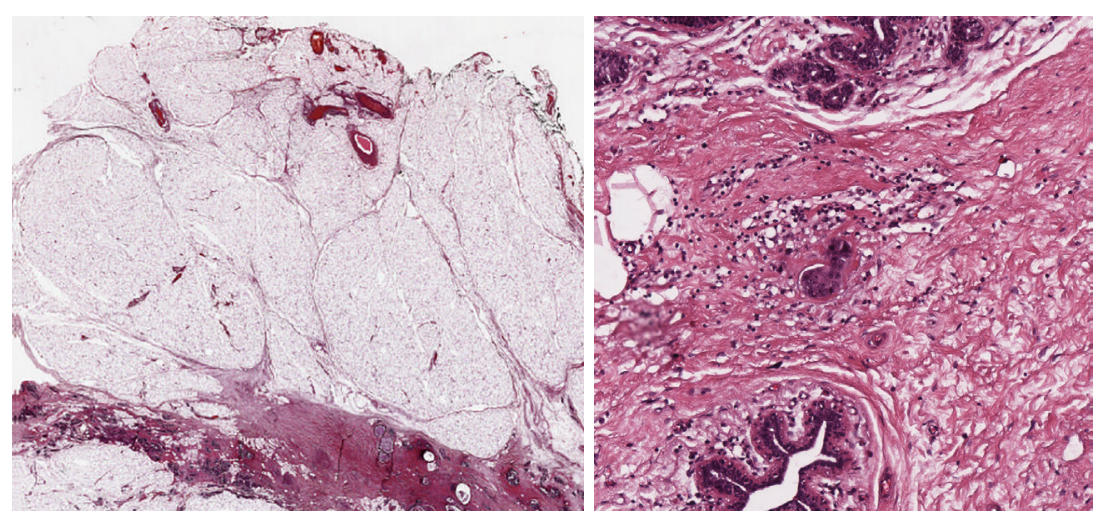

Figure 6 The present cases showed an apparently complete response to NAD on radiological and gross inspection. LS allowed to detect minute residual neoplastic foci (inset 10x) in the large fibrous area located in the tumour bed (magnification 1×). NAD, neo-adjuvant; LS, large section. 
The TE, defined as the tumor volume containing all the malignant structures, is the most important parameter influencing the choice of breast-conserving surgery (25). LS are useful also in determining the in situ and invasive component of a breast carcinoma. DCIS may not be identified in the dense as in fat replaced breast (19), a limit of human eye that can be avoided with LS. Especially in cases of invasive lobular carcinoma, parameters such as TS, type of growth, TE and resection margins are sometimes difficult to examine at naked eye, while they can be correctly defined in LS (22). LS have shown that in situ and invasive lobular carcinoma is a multifocal neoplastic lesion in more than $50 \%$ of the cases; poorly differentiated duct carcinoma in situ (DCIS grade 3) is frequently unifocal, whereas it is often multifocal when the in situ lesion is a welldifferentiated type (DCIS grade 1) (35).

LS shield the spatial configuration of tumor components and their relationship to resection margins, an essential parameter to be evaluated to obtain a curative excision, as the clinician may perform another surgical act or a radiation therapy if the tumor is present. LS allow a correct measurement of the distance between tumor and margins avoiding the concern raised by ink migration. LS has a pivotal role in histology reporting of breast specimens from patients who underwent neoadjuvant chemotherapy, used to convert inoperable to operable disease and to down-stage the disease, performing a wide local excision rather than mastectomy (32). Embedding the whole area previously occupied by the tumour (c.d. "tumour bed") improves the chance to detect small residual neoplastic foci, invisible at naked eye. Lesion distribution and disease extent represent prognostic parameters related to survival in breast carcinoma (12). Finally, vascular invasion also, a prognostic parameter used to plan chemotherapy, can be better identified with LS (36).

\section{Conclusions}

LS had proven their diagnostic utility in breast pathology. The technique has been invented more than one century ago, but it is still useful in clinical practice. It has been demonstrated that LS are cost effective and the TAT could be no more than 24 hours. Immunohistochemistry and molecular studies are also feasible. Due to all these reasons, more efforts are needed to use LS in daily routine practice.

\section{Acknowledgments}

Funding: None.

\section{Footnote}

Provenance and Peer Review: This article was commissioned by the Guest Editors (Gianluca Franceschini, Alejandro Martín Sánchez, Riccardo Masetti) for the series "Update of Current Evidences in Breast Cancer Multidisciplinary Management" published in Translational Cancer Research. The article has undergone external peer review.

Conflicts of Interest: All authors have completed the ICMJE uniform disclosure form (available at http://dx.doi. org/10.21037/tcr.2017.08.12). The series "Update of Current Evidences in Breast Cancer Multidisciplinary Management" was commissioned by the editorial office without any funding or sponsorship. The authors have no other conflicts of interest to declare.

Ethical Statement: The authors are accountable for all aspects of the work in ensuring that questions related to the accuracy or integrity of any part of the work are appropriately investigated and resolved.

Open Access Statement: This is an Open Access article distributed in accordance with the Creative Commons Attribution-NonCommercial-NoDerivs 4.0 International License (CC BY-NC-ND 4.0), which permits the noncommercial replication and distribution of the article with the strict proviso that no changes or edits are made and the original work is properly cited (including links to both the formal publication through the relevant DOI and the license). See: https://creativecommons.org/licenses/by-nc-nd/4.0/.

\section{References}

1. Cheatle GL. Clinical remarks on the early recognition of cancer of the breast. Br Med J 1906;1:1205-10.

2. Cheatle G. The relation between ducts and acini to cysts and cancer of the breast 21. Journal of Royal Society of Medicine 1914;7:214-44.

3. Cheatle GL. Cancer of the Breast: Treatment of the Proemial Breast. Br Med J 1922;1:869-71.

4. Wellings SR, Jensen HM. On the origin and progression of ductal carcinoma in the human breast. J Natl Cancer Inst 1973;50:1111-8.

5. Ingleby H HC. A method for the preparation of serial slices. Bullettin of the International Association of Medical Museums 1939;19:93-6.

6. Marcum RG, Wellings SR. Subgross pathology of the human breast: method and initial observations. J Natl Cancer Inst 1969;42:115-21.

7. Gallager HS, Martin JE. Early phases in the development 
of breast cancer. Cancer 1969;24:1170-8.

8. Gallager HS, Martin JE. The study of mammary carcinoma by mammography and whole organ sectioning. Early observations. Cancer 1969;23:855-73.

9. Sarnelli R, Squartini F. Multicentricity in breast cancer: a submacroscopic study. Pathol Annu 1986;21 Pt 1:143-58.

10. Tot T. DCIS, cytokeratins, and the theory of the sick lobe. Virchows Arch 2005;447:1-8.

11. Tot T. Clinical relevance of the distribution of the lesions in 500 consecutive breast cancer cases documented in large-format histologic sections. Cancer 2007;110:2551-60.

12. Tot T, Gere M, Pekar G, et al. Breast cancer multifocality, disease extent, and survival. Hum Pathol 2011;42:1761-9.

13. Faverly DR, Burgers L, Bult P, et al. Three dimensional imaging of mammary ductal carcinoma in situ: clinical implications. Semin Diagn Pathol 1994;11:193-8.

14. Going JJ, Moffat DF. Escaping from Flatland: clinical and biological aspects of human mammary duct anatomy in three dimensions. J Pathol 2004;203:538-44.

15. Moffat DF, Going JJ. Three dimensional anatomy of complete duct systems in human breast: pathological and developmental implications. J Clin Pathol 1996;49:48-52.

16. Tot T TL, Dean PB. Pratical Breast Pathology. Thieme, New York, NY, USA 2002.

17. Tot T, Tabar L. The role of radiological-pathological correlation in diagnosing early breast cancer: the pathologist's perspective. Virchows Arch 2011;458:125-31.

18. Tot $T$. The theory of the sick breast lobe and the possible consequences. Int J Surg Pathol 2007;15:369-75.

19. Tucker FL. Imaging-assisted large-format breast pathology: program rationale and development in a nonprofit health system in the United States. Int J Breast Cancer 2012;2012:171792.

20. Lega S, Malvi D, Flamminio F, et al. Applicazioni delle macrosezioni nella diagnostica del carcinoma mammario. Pathologica 2006;98:571.

21. Montironi R, Lopez Beltran A, Mazzucchelli R, et al. Handling of radical prostatectomy specimens: total embedding with large-format histology. Int J Breast Cancer 2012;2012:932784.

22. Tot T. Cost-benefit analysis of using large-format histology sections in routine diagnostic breast care. Breast 2010;19:284-8.

23. Hong NJ, Clarke GM, Yaffe MJ, et al. Cost-effectiveness analysis of whole-mount pathology processing for patients with early breast cancer undergoing breast conservation. Curr Oncol 2016;23:S23-31.

24. Jackson PA, Merchant W, McCormick CJ, et al. A comparison of large block macrosectioning and conventional techniques in breast pathology. Virchows Arch 1994;425:243-8.
25. Tot $\mathrm{T}$. The role of large-format histopathology in assessing subgross morphological prognostic parameters: a single institution report of 1000 consecutive breast cancer cases. Int J Breast Cancer 2012;2012:395415.

26. Tot T. Diffuse invasive breast carcinoma of no special type. Virchows Arch 2016;468:199-206.

27. Lynch SP, Lei X, Hsu L, et al. Breast cancer multifocality and multicentricity and locoregional recurrence.

Oncologist 2013;18:1167-73.

28. Asioli S, Morandi L, Cavatorta C, et al. The impact of field cancerization on the extent of duct carcinoma in situ (DCIS) in breast tissue after conservative excision. Eur J Surg Oncol 2016;42:1806-13.

29. Song SE, Seo BK, Cho KR, et al. Computer-aided detection (CAD) system for breast MRI in assessment of local tumor extent, nodal status, and multifocality of invasive breast cancers: preliminary study. Cancer Imaging 2015;15:1.

30. Pekar G, Hofmeyer S, Tabar L, et al. Multifocal breast cancer documented in large-format histology sections: longterm follow-up results by molecular phenotypes. Cancer 2013;119:1132-9.

31. Boros M, Voidazan S, Moldovan C, et al. Clinical implications of multifocality as a prognostic factor in breast carcinoma - a multivariate analysis study comprising 460 cases. Int J Clin Exp Med 2015;8:9839-46.

32. Pinder SE, Provenzano E, Earl H, et al. Laboratory handling and histology reporting of breast specimens from patients who have received neoadjuvant chemotherapy. Histopathology 2007;50:409-17.

33. Provenzano E, Bossuyt V, Viale G, et al. Standardization of pathologic evaluation and reporting of postneoadjuvant specimens in clinical trials of breast cancer: recommendations from an international working group. Mod Pathol 2015;28:1185-201.

34. Bossuyt V, Provenzano E, Symmans WF, et al. Recommendations for standardized pathological characterization of residual disease for neoadjuvant clinical trials of breast cancer by the BIG-NABCG collaboration. Ann Oncol 2015;26:1280-91.

35. Foschini MP, Flamminio F, Miglio R, et al. The impact of large sections on the study of in situ and invasive duct carcinoma of the breast. Hum Pathol 2007;38:1736-43.

36. Foschini MP, Baldovini C, Ishikawa $Y$, et al. The value of large sections in surgical pathology. Int J Breast Cancer 2012;2012:785947.

Cite this article as: Monica MA, Morandi L, Foschini MP. Utility of large sections (macrosections) in breast cancer pathology. Transl Cancer Res 2018;7(Suppl 3):S418-S423. doi: 10.21037/tcr.2017.08.12 